\title{
Dietary patterns and the risk of incident CVD in Scottish adults
}

\author{
G. McNeill, L. C. A. Craig, N. Sarwar and A. Sullivan-Higham \\ Public Health Nutrition Research Group, Division of Applied Health Sciences, University of Aberdeen, \\ Aberdeen AB25 2ZD, UK
}

The aim of this study was to investigate associations of dietary patterns with incident CVD outcomes in Scottish adults using the Scottish Health Surveys. These are large, nationally representative cross-sectional surveys of lifestyle and health conducted in 1995 , 1998 and 2003. The data from the three surveys were combined and the dietary data re-coded to harmonize the food groups and intake categories across the surveys where necessary. Principal components analysis of the dietary data identified two dietary patterns: a 'healthy' pattern (loading positively for fruit, high fibre breakfast cereals, vegetables, wholemeal bread, potatoes, pasta and rice and oily fish, and negatively for white bread and salt use), and an 'unhealthy' pattern (loading positively for cakes, confectionery, white bread, cheese, soft drinks and meat and negatively for low-fat milk and low-fat spread). These patterns accounted for $11 \%$ and $9 \%$ of the total variance in the dietary data, respectively. Morbidity and mortality data on all participants up to the end of 2007 were obtained by record linkage to the Scottish Morbidity Records, which was carried out by Information Services Division Scotland. CVD outcomes were defined as non-fatal hospital admissions or death coded with International Classification of Disease revision 10 (ICD 10) codes I20-I25, I60-I69 and G45. Time to event analyses were conducted using Cox's regression with progressive adjustment for age, sex, socio-economic status, physical activity, smoking, alcohol intake and BMI.

In 13226 participants aged 35-90 years without CVD or diabetes at baseline, there were 901 incident CVD outcomes during a mean follow-up of 8.3 years. Participants in the highest quarter of the score for the 'healthy' diet pattern had a lower risk of CVD than those in the lowest quarter (see Table). Although there was some attenuation in the association upon adjustment for other lifestyle factors, particularly socio-economic status and smoking, it remained statistically significant $(P=0.016)$ in the fully adjusted model. By contrast, corresponding hazard ratios for CVD associated with the 'unhealthy' diet pattern were not statistically significant in any of the analyses. Similar results were obtained when analyses were restricted to 633 coronary heart disease outcomes (ICD 10 codes I20-I25).

\begin{tabular}{lcc}
\hline & \multicolumn{2}{c}{ Hazard ratio (95\% CI) for CVD in top quarter $v$. bottom quarter } \\
\cline { 2 - 3 } Adjusted for & 'Healthy' diet pattern & 'Unhealthy' diet pattern \\
\hline Age and sex & $0.50(0.42,0.61)$ & $1.18(0.97,1.43)$ \\
As above plus socio-economic status & $0.61(0.50,0.74)$ & $1.14(0.94,1.38)$ \\
As above plus physical activity & $0.65(0.54,0.79)$ & $1.13(0.93,1.37)$ \\
As above plus smoking & $0.79(0.64,0.96)$ & $1.09(0.90,1.32)$ \\
As above plus alcohol intake & $0.78(0.64,0.96)$ & $1.07(0.88,1.30)$ \\
As above plus BMI & $0.78(0.64,0.96)$ & $1.10(0.91,1.34)$ \\
\hline
\end{tabular}

In 8061 participants with available information from biochemical and biophysical measurements, the mean HDL-cholesterol was $0.14 \mathrm{mmol} / \mathrm{l}$ higher, mean non-HDL-cholesterol was $0.12 \mathrm{mmol} / \mathrm{l}$ lower and mean fibrinogen was $0.11 \mathrm{~g} / \mathrm{l}$ lower among individuals in the top quarter of the 'healthy' diet pattern compared to those in the bottom quarter (all $P<0.001$ ). By contrast, there were no significant differences in the levels of total cholesterol or systolic or diastolic blood pressure (all $P>0.4$ ). These results indicate a modest inverse association between 'healthy' diet pattern and CVD risk, possibly mediated through beneficial effects on circulating concentrations of lipid fractions and inflammatory markers. 